\title{
Relación entre ejemplarismo y cultura en la concepción agustiniana de la historia*
}

Desde Platón a Edm. Husserl la inteligencia humana ha sentido una atracción insistente hacia las "ideas ejemplares". Ortega y Gasset considera el día más feliz para la filosofía aquel en que estas ideas fueron pensadas por Platón. Esto dicho es más de admirar si se tiene en cuenta que el pensamiento orteguiano, ligado a la vida como realidad histórica, sigue una directriz opuesta a la mentalidad platónica. Pese a ello, reconoce el pensador español la imprescindible necesidad que tiene la mente humana de eso paradigmas que le atraen y le fascinan. Como puntos luminosos, como estrellas de luz en el firmamento de la propia conciencia.

Para Platón las ideas ejemplares son respecto de la realidad sensible y cambiable, la verdadera ousia, en tal manera que aquélla, la realidad sensible, es tanto más perfecta cuanto mejor imita y participa de la idea paradigmática, fuente última de todo verdadero ser. Son también logos en el doble sentido de ser algo inteligible y lo único capaz de dar inteligibilidad a las realidades transitorias. Finalmente, son telos en cuanto que atraen al ser contingente y mudable a su imitación, en tal manera que éste será tanto más perfecto cuanto mejor realice dicha imitación.

De esta su visión metafísica deduce Platón la necesidad de una cultura unitaria y la negación radical del valor de la historia. Según él la cultura, expresión temporal y sensible de los valores eternos del mundo paradigmático tiene que ser forzosamente una. Pues sólo hay una posibilidad única de imitación perfecta de estos valores. Para Platón no cuentan las virtualidades psicológicas de los individuos y de los pueblos. Sólo tiene valor en el mundo sensible lo que sea reflejo del eterno modelo ideal. Por eso, en buena lógica, siente entusiasmo en el

\footnotetext{
* Este estudio fue presentado al XIV Congreso Internacional de Filosofía celebrado en Viena, 2-9 de septiembre de 1968.
} 
campo del arte por el hieratismo egipcio, expresión máxima de lo inmutable y eterno en una realización sensible.

La historia, a su vez, perecedera y caduca, pura contingencia y aabilidad, mundo de sombras, no puede incluir en sí misma un auténtico valor. Tanto será menos imperfecta cuanto más tienda a reproducir los eternos arquetipos ideales.

Hemos recordado esta enseñanza de Platón porque la creemos un necesario punto de partida para comprender con hondura la actitud de San Agustín. El doctor cristiano sigue de cerca al pensador griego. Pero al mismo tiempo hay que subrayar su potente originalidad por la que so diferencia del viejo maestro de Atenas.

La primera nota que distingue netamente el pensamiento de San Agustín del de Platón es la tendencia de aquél a personificar el cosmos ideal paradigmático de éste. Para San Agustín no es este mundo ideal un valor en sí, una realidad plena y perfecta. Ve los paradigmas de las cosas en la Trinidad creadora, que va dejando huellas de su vida trinitaria en los elementos ternarios de lo creado. San Agustín acepta con entusiasmo el "cosmos noetos" de Platón. Pero en vez de buscar para este cosmos un lugar fuera del mundo sensible, lo integra a su visión personalista de la Trinidad. La segunda persona, el Logos sustancial, es el lugar celeste del mundo paradigmático de Platón.

De esta visión ejemplarista de la Trinidad tenemos que partir para comprender las repercusiones del ejemplarismo agustiniano en la interpretación de la historia y de la cultura. Se ha dicho reiteradamente por los comentarios de San Agustín que su concepción trinitaria no es algo adicional a su filosofía, sino que entra en la constitución de la misma. Es esto lo que intentaremos hacer ver a lo largo de nuestro estudio.

San Agustín, que ha sido llamado el primer hombre moderno, siente ya con hondura los problemas de la historia. En ello hallamos una segunda nota que la diferencia de su maestro Platón. Si para éste, como terminamos de afirmar, la historia cae íntegramente del lado de la noche tenebrosa de lo cambiable, San Agustín la estima tanto que hace de ella el objeto de la reflexión más profunda y duradera de su vida. Su obra De Civitate Dei es el fruto de esta reflexión.

Es en esta obra donde San Agustín estudia la concepción ejemplarista aplica a la historia. En uno de sus libros centrales nos hace ver cómo la Trinidad va dejando huellas de su presencia en la creación. Esta presencia se halla del modo más admirable en el alma humana, "la más 
cercana a Dios, por naturaleza, de todas las creaturas" ". Somos, conocemos que somos y amamos este ser y este conocer, dice textualmente San Agustín. Y en estas propiedades del alma humana ve un reflejo del divino ejemplar, que es la misma Trinidad Augusta.

San Agustín halla en este ejemplarismo, aplicado al alma humana, la clave de la historia íntima de cada hombre. Esta historia cuando intenta alcanzar la perfección, busca en la imitación de la Trinidad la meta de sus aspiraciones. En el libro de las Confesiones San Agustín ha descrito sus esfuerzos para llegar a esta meta desde la noche del pecado.

Pero si las Confesiones es la historia del alma de San Agustín, repetida incontable número de veces por otras almas, la historia de la ( iudad de Dios es la historia de la humanidad peregrina. Y también es el ejemplarismo una doctrina clave para interpretar la marcha grandiosa de la Ciudad de Dios peregrina.

De Civitate Dei, en un pasaje de excepcional importancia, nos muestra el maravilloso panorama metafísico de su visión ejemplarista. En dicho pasaje, establece como principio general que la Trinidad es intima " todas sus obras. Muy luego hace aplicación de este principio a la Ciudad de Dios, afirmando de ella que en la Trinidad tiene su origen, forma y felicidad ${ }^{2}$.

Para comprender con hondura esta triple relación de la Ciudad de Dios con la Trinidad, tenemos que recordar el triple tema en torno al cual gira todo el pensamiento agustiniano. Se le ha resumido en tres palabras: sum, cognosco, amo. Estas palabras sintentizan los tres grandes problemas humanos: el problema del ser y de la existencia, el problema de la verdad y el problema de la felicidad en el amor.

En este pasaje nos dice el doctor africano que el ser de la Ciudad de Dios proviene de la Trinidad que la fundó. Que su conocimiento sumo, es decir, su sabiduría, dimana de la Trinidad que la ilumina. $\mathrm{Y}$ que su felicidad no puede ser otra que gozo de Dios. De esta suerte la Ciudad Santa, sigue razonando San Agustín con expresiones muy consisas: Subsistiendo, se modifica. Contemplando, se ilumina. Uniéndose a Dios, goza de El. La primera frase es una hondísima expresión con la que se quiere echar un puente entre el platonismo, ajeno a la historia, y las exigencias de ésta. La segunda subraya cómo la verdad

De Civitate Dei XI, 26, PL. 41.

Ibid. XI, 24, PL. 41. 
eterna irradia claridad en las almas que la miran y contemplan. La tercera afirma netamente que la plenitud de vida y la meta de la historia no es otra que la fruición de lo divino, fruición que la Ciudad de Dios ya anticipa en alguna manera en su peregrinación terrena.

Siguiendo su razonamiento trinitario, formulado en ternas de extraordinaria precisión, concluye San Agustín, hablando siempre de la Ciudad de Dios: "Existe, ve y ama. Vive en la eternidad de Dios, brilla en la verdad de Dios y goza en su bondad" 3 .

De su visión ejemplarista infiere San Agustín las virtudes fundamentales que deben adornar a la Ciudad Santa. Contrapone estas virtudes a las de la ciudad de Roma, el paradigma histórico de la ciudad terrena. Con palabras concisas, pero muy expresivas escribe: "La ciudad de arriba es incomparablemente más luminosa (que Roma). En ella, la unctoria es la verdad, el honor es la santidad, la paz es la felicidad y la vida es la eternidad" 4 .

Adviértase la antitesis que establece entre las virtudes paradigmáticas de la Ciudad de Dios y Roma. Roma, volcada fuera de sí, busca la victoria sobre sus enemigos. La Ciudad de Dios, vuelita sobre sí, busca la eterna verdad que la ilumina. Roma pone su dignidad y su honor en triunfar sobre los otros pueblos. La Ciudad de Dios pone su más alta dignidad en su propia santidad. Roma anhela una paz terrena. La Ciudad de Dios una paz que es la plena felicidad. Roma quiere vivir eternamente. La Ciudad de Dios participa de la eternidad de Dios.

Detengámonos en la exposición de estas cuatro virtudes paradigmáticas de la Ciudad de Dios, que nos dan la clave de la íntima estructura de su historia. Incontables veces nos dice San Agustín que esta ciudad es peregrina. Es decir: va camino de un fin escatológico. Pero aunque peregrina, refleja ya en su peregrinación estas cuatro virtudes que son la cuádruple columna sobre la que se asienta la vida íntima de esta Ciudad, de sentido exclusivamente ético, no político, y a la que pertenece todo hombre de buena voluntad.

La primera virtud paradigmática que señala San Agustín es la verdad. Sobre este tema hoy se halla la filosofía muy lejos de su modo

3 Ibid., l. c.

4 Ibid. II, 29, PL. 41. El esquema trinitario no se mantiene en esta ocasión, pero sí el sentido ejemplarista. Ello quiere decir que los esquemas agustinianos no son rígidos. $Y$ aunque su tendencia es constante al esquema ternario, en esta ocasión ha desdoblado lo apropiado al Espíritu Santo en dos virtudes, santidad y felicidad. Ello no merma valor a su ejemplarismo trinitario. 
de ver. Después de largos siglos en búsqueda de la verdad, la filosofía se ha declarado más problemática que adquisitiva, más en camino, que en estancia iluminada por el sol de la verdad. San Agustín no pensaba así. En sus Confesiones suspira por la verdad: "O veritas, veritas, quam intime etiam tum medullae animi mei suspirabant tibi" ${ }^{5}$. En De Civitate $D e i$, donde se trata de la historia de la humanidad, vuelve sobre el tema para lamentar la multitud de opiniones contradictorias en la ciudad del mal. Por tal motivo, escribe, se la ha llamado místicamente Babilonia, "es decir, Confusión ${ }^{6}$.

Frente a ella San Agustín ensalza a la Ciudad de Dios, asentada en ia verdad, la que tiene por filósofos a los auténticos amadores de la sabiduría, y por guías, a sabios y doctores en probidad y piedad.

Desde el punto de vista de la filiación de las ideas es de advertir que esta postura agustiniana tiene afinidad con la del viejo maestro Platón. En Las Leyes conceptúa imposible el pensador griego que la ciudad pueda establecerse de un modo consistente sin la verdad. Y hasta podemos añadir que uno y otro ven este problema desde el ejemplarismo. Ambos, en efecto, miran a Dios como fuente de toda verdad. Con esta diferencia, sin embargo: que si para Platón Dios es la medida del hombre, para San Agustín la medida y paradigma del hombre es el misterio cristiano de la Trinidad. La segunda Persona de este misterio, el Logos, ha señalado al hombre los caminos de la verdad. Hasta llegar un día a aparecer vestido de carne entre los hombres. Ello mótiva un nuevo contraste con el platonismo. Para éste la vestimenta carnal del Logos fue siempre un motivo de escándalo. San Agustín tomó conciencia de él. Pero, en lugar de asentir a la escuela que ama, la impugna decididamente. San Agustín ve en la Encarnación la suprema dignación de la Verdad, que se hace sensible para que el hombre más fácilmente la pueda aceptar y seguir ${ }^{7}$.

Pese a estas diferencias tan sustanciales. San Agustín con Platón y el platonismo considera la verdad como la piedra angular del místico edificio de la Ciudad de Dios. Y esta verdad es un reflejo de la verdad eterna que en la Encarnación del Logos se hizo sensible y humana.

La segunda virtud paradigmática que señala San Agustín es la santidad. Con ello se anticipa a uno de los más graves problemas de

5 Confesiones III, 6 PL.

6 De Civitate Dei XVIII, el PL. 41.

7 Tema central en toda la obra, especialmente es desarrollado en el libro X. 
nuestra hora. Hoy la mentalidad marxista subraya que la clave de la vida ética de la sociedad se halla en las estructuras de la misma. Estructuras de opresión a lo largo de los siglos y que ahora deben ser radicalmente superadas para dar paso a estructuras socialistas. Esta mentalidad, como atmósfera espiritual diluída, se la siente hoy doquier.

San Agustín ya toma conciencia del problema de las estructuras y de su influjo en la vida de los pueblos. Pero lo resuelve de modo distinto al que terminamos de aludir. Para San Agustín el problema primario para toda ciudad y toda república ${ }^{8}$ no es el de las instituciones de.las estructuras, sino el de las costumbres. Roma sucumbe porque antes de que los bárbaros asaltan sus muros, ya se habían desmoronado las buenas costumbres. Y es inútil que los muros se mantengan en pie, si se viene abajo el edificio de la moralidad pública.

- Con tal exigencia ha dado importancia San Agustín al tema moral que ha suscitado una disputa entre sus expositores sobre si conceptuaba a Roma como república legítima. La dificultad estriba en que para San Agustín no hay república donde no se halla vigente la justicia. Ahora bien; al echar en cara a Roma sus múltiples crímenes, da pie para preguntarse si siempre la conceptuó verdadera república ${ }^{9}$.

No es ciego, sin embargo, San Agustín para las virtudes naturales de los romanos. Por ellas Roma alcanzó su dignidad y su honor. De tales virtudes hizo el mejor encomio al proponerlas como ejemplos a los peregrinos de la Ciudad de Dios. "Diligenter et sobrie illa intueantur exempla", escribe San Agustín ${ }^{10}$. Cierto que ve estos ejemplos afeados por la escoria de la ambición. Pero no obstante ello, los santos de la Ciudad de Dios deben hallar un estímulo para el heroismo en las acciores magnánimas de los romanos.

En este razonamiento de San Agustín queda bien patente, por una parte, la importancia decisiva de las virtudes morales para lo constitución de las sociedades humanas. Y por otra, cómo abre la puerta el humanismo cristiano, al subrayar las virtudes naturales de los romanos como ejemplos que deben ser imitados por los mismos santos de la Ciudad de Dios.

Pero este humanismo cristiano que mira con complacencia los valores y virtudes de aquí abajo, no le impide a San Agustín ver siempre

8 De Civitate Dei II, 2 PL.

9 Ibid. II, 21 y 25 PL.

10. Ibid. V, $18 \mathrm{PL}$. 
tl modelo último de toda verdadera santidad en el misterio trinitario, y especialmene en la segunda Persona, hecha carne para sernos ejemplo vivo de santidad. Muy por encima de los modelos humanos, afeados por múltiples defectos, San Agustín propone como modelo a Cristo, mediador entre Dios y los hombres y, al mismo tiempo, modelo y ejemplar de todos.

La tercera virtud paradigmática que menciona San Agustín es la paz de la felicidad. A ella vincula la eternidad. No puede haber felicidad perfecta si ésta es algo meramente personal y transitorio. "De beatitudine pacis aeternae", es el tema de uno de los capítulos de su gran obra ${ }^{11}$. La paz eterna, vigente siempre en la Ciudad de Dios, es un reflejo de la paz en que vive el misterio trinitario, paradigma eterno de toda verdadera paz.

Podemos disinguir dos momentos en el análisis agustiniano de la paz. En un primer momento San Agustín tiende su vista por todo el cosmos y advierte en todos los seres un deseo inextinguible de paz, a la que define de un modo maravilloso e insuperado: "tranquillitas ordinis". ${ }^{12}$. Se detiene con morosa complacencia en describir cómo todos los seres en gradación ascendente buscan la paz dentro de un orden. El cuerpo busca la paz en la ordenada complexión de sus partes. El alma irracional en la ordenada calma de sus apetitos. El alma racional en la ordenada armonía entre el conocimiento y la acción. La paz entre Dios y el hombre brota de la ordenada obediencia por la fe bajo la ley eterna. Hasta lo más impensable, la guerra feroz y mostruosa no tiene otro fin que la paz. "Hanc "pacem requirunt laboriosa bella", escribe con frase lapidaria el doctor africano ${ }^{13}$.

Nos parece que en esta concepción cósmica de la paz San Agustín está influído tanto por la visión filosófica de los estoicos, como por la realización política del "ordo romanus". San Agustín, abierto a todo sano influjo, no se desinteresa de ningún elemento cultural valioso. Pero lo más importante y decisivo es siempre su visión ejemplarista por la que fija su mirada en la paz eterna y desde ella enjuicia la paz en este mundo.

Este primer momento de la visión cósmica de la paz en San Agustín adquiere mayor plenitud al aplicarse a las sociedades humanas. Mas en este momento de su análisis San Agustín ya no se contenta con la pers-

11 Ibid. XIX, II PL.

Ibid. XIX, $13 \mathrm{PL}$.

Ibid. XV, $4 \mathrm{PL}$. 
pectiva del orden. Recurre a otro vocablo de más intimidad humana y habla de la concordia. Una sentencia agustiniana nos introduce de lleno en este tema. Dice así: "Nihil enim est quam hoc genus (hominum) tam discordiosum vitio, tam sociale natura" ${ }^{14}$. Merece esta sentencia un breve comentario.

El filósofo de la historia, Ortega y Gasset, ha protestado de la afirmación reiterada que define al hombre como animal social. Es sabido que esta definición viene de Aristóteles, quien llama al hombre "zoon politikon". Ortega y Gasset protesta porque, si bien es cierto que hay en el hombre tendencias hacia la más pura sociabilidad, también hay otras de racional tendencia contraria. Fuerzas disolventes que amenazan destruir de continuo toda convivencia humana. También a San Agustín preocupó el problema y lo resolvió en la frase precisa que hemos citado. Según ella lo que disocia es el vicio. Pero el hombre por naturaleza como obra de Dios, es un ser social.

Desde esta visión de la naturaleza humana y del influjo disolvente del vicio adquieren luminosidad los dichos de San Agustín en los que afirma que la paz de los hombres entre sí consiste en su ordenada concordia. Que la paz de la cosa es la ordenada concordia entre los que mandan y los que obedecen. Que la paz de la ciudad es la ordenada concordia entre los ciudadanos que gobiernan y los gobernados. Finalmente, que la paz de la ciudad celestial es la unión ordenadísima y concordísima para gozar de Dios y a la vez en Dios ${ }^{15}$.

Cuán bellos en su expresión y qué hondo contenido encierran los dos epítetos, "ordinatissima et concordissima", con los que San Agustín adjetiva la paz de la Ciudad Santa. Expresan en fórmula insuperable el ideal de la más elevada cultura de la paz.

Quedaría, con todo, incompleta esta visión agustiniana de la paz si no se le vinculara a la eternidad. Es cierto que la eternidad, tal como ia prospecta San Agustín tiene siempre un sentido escatológico. Pero es que el pensamiento agustiniano es incomprensible sin esta evocación. $Y$ 'desde ella hay que afirmar que nadie es plenamente felliz si siente amenazada su felicidad presente. Por ello, la felicidad de la Ciudad de Dios sólo adquiere pleno sentido en vinculación con la eternidad escatológica. Las generaciones humanas, escribe, no son ni imagen siquiera de la eternidad. Se asemejan al árbol de hoja perenne, que parece conser-

\footnotetext{
14 Ibid. XII, 27 PL.

15 Ibid. XIX, 13 PL.
} 
var siempre el mismo verdor, en tanto que van cayendo unas hojas y brotando otras, perpetuándose así la apariencia de su frescor ${ }^{16}$.

Parece recoger en estas frases San Agustín ell pensamiento de Aristóteles cuando afirma que por medio de la generación los seres naturales imitan la eternidad de Dios. Pero esta deficiente inmortalidad, propugnada por más de un sistema fillosófico moderno, no es suficiente para el jensamiento agustiniano, que pide la eternidad personal para cada uno de los moradores de la Ciudad de Dios. En ella, todos serán inmortales.

Después de este canto a la paz, a San Agustín no le resta más que escribir el último capítulo de su gran obra, al que titula: De aeterna felicitate sabbatoque perpetuo ${ }^{17}$. Es esta eterna felicidad y este sábado perpetuo la piedra clave que cierra el edificio de la paz, construído por San Agustín desde su concepción ejemplarista como interpretación última de la historia.

De su visión ejemplarista fluye también su visión honda de la cultura humana. Con Platón mantiene San Agustín la unidad de la cultura. Pero esa unidad la limita a los elementos esenciales.. No puede haber auténtica cultura humana si no hay verdad, santidad, felicidad y paz eterna. No obstante, estas virtudes paradigmáticas no ponen óbice, según San Agustín, al desarrollo del espíritu de los diversos pueblos. Lenguas, costumbres, instituciones, procesos históricos : todo tiene un valor mienras no se opongan a las exigencias esenciales de la única cultura.

San Agustín tiene sobre este problema una extraordinaria luminosidad para aquel tiempo. Mientras los estoicos poilarizaban su atención bacia un cosmopolitismo abstracto, carente del jugo vital que dimana de Ias íntimas esencias de los diversos pueblos, San Agustín, más iluminado, puso las bases de la verdadera filosofía de la cultura con estas palabras, dignas de ser hoy recordadas: "La ciudad celestial, durante su peregrinación, va llamando ciudadanos por todas las naciones y formando de todas las lenguas, de costumbres, y de instituciones... Ella no suprime ni destruye nada, antes bien, lo conserva y acepta, y ese conjunto, aunque diverso en las diferentes naciones, se flecha, con todo, a un único y mismo fin, la paz terrena, si es que no se opone a la religión que enseña el culto ủe Dios, único y verdadero. En su viaje la ciudad cellestial usa también de la paz terrena y de las cosas necesariamente relacionadas con la condición actual de los hombres. Protege y desea el acuerdo de quereres

16 Ibid. XXII, 1 PL.

17 Ibid. XXII, $30 \mathrm{PL}$. 
entre los hombres cuanto es posible, dejando a salvo la piedad y la religión, y supedita la paz terrena a la paz cellestial" ${ }^{18}$.

Dada la brevedad que impone la comunicación, dejamos al lector $\epsilon 1$ comentario de este bellísimo texto. Bástenos dejar constancia de cómo el ejemplarismo agustiniano no ha ahogado la legítima comprensión de las culturas. Al contrario; su mirada ail eterno modelo ha iniciado a San Agustín a examinar las incontables riquezas en que puede ofrecer el germen divino, sembrado en el espíritu de los puebilos por el Logos.

18 Ibid. XXII, 17 PL.

Enrique Rivera de Ventośa 